Rev. salud pública. 13 (6): 966-979, 2011

\title{
Formación de médicos y enfermeras para la detección temprana del cáncer de mama en México
}

\section{Doctors and nurses' training for the early detection of the breast cancer in Mexico}

\author{
María C. González-Robledo ${ }^{1}$, Luz M. González-Robledo ${ }^{2}$, Marta Caballero ${ }^{3}$ y \\ Matilde E. Aguilar-Martínez ${ }^{4}$
}

\begin{abstract}
1 Centro de Investigación en Sistemas de Salud, Instituto Nacional de Salud Pública. México. cecilia. gonzalez@insp.mx

2 Facultad de Medicina de la Universidad Autónoma del Estado de Morelos, México. luz.gonzalez@, uaem.mx

3 Instituto Profesional de la Región Oriente, Universidad Autónoma del Estado de Morelos, México. martacg@uaem.mx

4 Dirección de Innovación de Servicios y Sistemas de Salud. Instituto Nacional de Salud Pública. México. elizabeth.aguilar@insp.mx
\end{abstract}

Recibido 18 Marzo 2011/Enviado para Modificación 7 Diciembre 2011/Aceptado 15 Diciembre 2011

\section{RESUMEN}

Objetivo Analizar la formación académica que reciben los estudiantes de medicina y enfermería sobre el cáncer de mama (CaMa) durante el trascurso de su carrera universitaria.

Materiales y Métodos Estudio exploratorio realizado en 2009. Basado en entrevistas semi-estructuradas a actores clave y revisión documental. Población de estudio: 199 Facultades/Escuelas de medicina y 108 Escuelas/Institutos de enfermería. Unidad de análisis: plan de estudios.

Resultados En 16,8\% de los planes de estudio de medicina y 6,3\% de enfermería se encontró información específica de contenidos sobre CaMa. La capacitación que reciben los médicos es predominantemente desarrollada por las áreas biomédica y clínica y en menor proporción en el área socio-médica. En enfermería los temas que más se desarrollan son en áreas comunitarias, abordando contenidos de promoción de la salud y prevención de la enfermedad (estilos de vida saludable e identificación de mujeres con alto riesgo de padecer CaMa).

Discusión Se encontró escasa evidencia sobre entrenamiento específico para la detección y atención del cáncer de mama en los planes de estudio. Por los resultados encontrados es necesario fortalecer los currículos para formar a los futuros profesionales con capacidades para trabajar en la solución de los problemas y necesidades específicos de salud de la población particularmente en acciones de prevención primaria y secundaria. 
Palabras Clave: Formación de recursos humanos, personal de enfermería, cuerpo médico, neoplasias de la mama (fuente: DeCS, BIREME).

\section{ABSTRACT}

Objective Analyzing formal knowledge about breast cancer $(\mathrm{BC})$ issues that medical and nursing students receive from different syllabuses and courses during their undergraduate studies.

Materials and Methods This exploratory study was conducted in 2009; it was based on semi-structured interviews applied to key stakeholders and complemented by an analysis of their undergraduate courses. The study population consisted of 199 Faculties and Schools of Medicine and 108 Schools and Colleges of Nursing and the unit of analysis consisted of the syllabuses for the undergraduate courses.

Results $16.8 \%$ of the medical courses and $6.3 \%$ of the nursing courses had specific information about BC. Medical students predominantly received their training in biomedical and clinical areas and the socio-medical area to a lesser extent. The issues in nursing courses was mainly focused (in community areas) on promoting health and preventing disease (healthy lifestyles and recognition of women at high risk regarding $\mathrm{BC}$ ).

Discussion Little evidence was found that medicine and nursing syllabuses contain issues related to BC. Medical and nursing curricula should be strengthened to improve professional skills and abilities related to BC to solve the affected population's problems and health needs, particularly regarding primary and secondary prevention.

Key Words: Professional education, nursing personnel, physician, breast cancer (source: MeSH, NLM).

$\mathrm{E}$ 1 cáncer de mama es el tipo de cáncer más común en las mujeres independientemente de su raza o grupo étnico (1). Estimaciones realizadas por la American Cancer Society indican que se presentaron 1301867 casos nuevos diagnosticados y 464854 muertes en el año 2007 por esta causa en el mundo (2). Con relación a la atención de esta enfermedad se sabe que la detección temprana combinada con un tratamiento adecuado es la estrategia más eficaz para reducir la mortalidad por esta causa (3).

También se ha demostrado que la mejor forma de hacer detección temprana del cáncer de mama es a través de un programa organizado para tamizar aspectos mamográficos en la población $(4,5)$. Por el contrario, existe evidencia muy controversial sobre la eficacia de la detección temprana con programas basados en la autoexploración mamaria y el examen clínico de 
los senos (6-8). No obstante en países con ingresos limitados, en donde no existe la posibilidad de tener un programa para tamizar en la población, son muy útiles estos procedimientos, ya que se han observado beneficios muy significativos como la reducción del tiempo entre la detección y la visita a un médico, y como consecuencia, la detección del cáncer de mama en fases más tempranas. Es necesario señalar que esto es posible mientras que exista personal entrenado para realizar el examen clínico y haya una adecuada capacitación de las mujeres para hacerse la autoexploración (9$11)$.

El primer contacto de las mujeres con el personal de salud - médicos y enfermeras- puede influir de manera directa, en la aplicación de medidas tendientes a conseguir una detección temprana del cáncer de mama. Esto por su capacidad de compartir información de calidad y realizar un trabajo educativo sistemático para que la población femenina adquiera conocimientos y adopte actitudes y conductas responsables con relación al auto cuidado de la salud de la mama, además de realizar procedimientos tendientes a detectar algún tipo de anormalidad en los senos mediante una buena historia clínica y un examen clínico de mamas realizado por personal bien capacitado $(12,13)$.

En México, el panorama epidemiológico de los dos principales cánceres femeninos muestra dos tendencias muy claras. En 2006 la tasa de mortalidad por CaMa superó a la de cáncer cervico-uterino por primera vez en la historia (14). Además la tendencia del primero es a incrementarse mientras que la del segundo tiende abiertamente a la reducción (15). Aún cuando no existe una evidencia contundente, se cree que la disminución que muestra el cáncer de cérvix está relacionada con la política gubernamental iniciada en los años noventa para hacerle frente, donde se incluyen tanto medidas preventivas como curativas. Para el cáncer de mama en cambio, no existe todavía una respuesta articulada e integral por parte del sistema de salud. La sensibilización de los actores públicos es todavía temprana a pesar de ser un tema con el que las organizaciones de la sociedad civil han trabajado ya durante algunos años. La evidencia epidemiológica obliga a una respuesta inmediata.

En el país se ha documentado que la falta de oportunidad en la detección temprana del cáncer de mama es debida, en parte, a la escasez 
de personal médico y paramédico entrenado, particularmente en personal de primer contacto (16-18). Adicionalmente, es importante destacar que la información disponible en el país sobre el tema de recursos humanos y cáncer de mama es muy reducida.

El propósito del artículo es presentar un panorama general del entrenamiento que reciben los estudiantes de medicina y enfermería sobre el cáncer de mama durante el trascurso de la carrera, con vistas a formular recomendaciones a las autoridades académicas que conduzcan a mejorar la formación y entrenamiento de este personal en acciones de promoción de la salud y detección temprana para el cáncer de mama en México.

\section{METODOLOGÍA}

Se realizó estudio exploratorio transversal durante el año 2009, en el cual se recolectó y analizó información basada en dos tipos de técnicas: análisis documental para obtener información sobre la formación y entrenamiento de estudiantes de medicina y enfermería (a nivel de licenciatura) en cáncer de mama en México; y entrevistas semiestructuradas a personal académico, que buscaban identificar y clasificar el tipo y volumen de material didáctico utilizado por las diversas escuelas y facultades de medicina y enfermería para brindar dicho entrenamiento.

El directorio de las escuelas y facultades de medicina y enfermería (a nivel universitario) se construyó tomando como base el registro de Universidades e Institutos Tecnológicos de la Asociación Nacional de Universidades e Instituciones de Educación Superior (ANUIES) (19), organización que agremia a las principales instituciones de educación superior del país tanto públicas, como privadas y que atienden al $80 \%$ de la matrícula de alumnos que cursan estudios de licenciatura y de posgrado. Este catálogo proporcionó información académico-administrativa de cada uno de los programas de medicina y enfermería ofertados en la totalidad de entidades federativas del país.

La búsqueda de los planes curriculares se hizo en las páginas Web de las universidades identificadas en el directorio. Se encontró este tipo de información con los siguientes datos: créditos que otorga cada ciclo o unidad didáctica, duración y periodicidad de los programas (años, 
semestres, trimestres) y, en algunos casos, mayor detalle sobre los objetivos, propósitos y características específicas de los programas.

La población de estudio estuvo constituida por 199 Facultades/Escuelas de medicina y enfermería de las cuales 128 fueron públicas y 71 privadas. De todas ellas, 91 correspondieron a Facultades/Escuelas de medicina y 108 de enfermería. La unidad de análisis la constituyó el plan de estudios. En ellos se buscaron los siguientes aspectos: título que otorga, año del plan de estudios, asignaturas relacionadas con cáncer de mama, semestres/ ciclos/módulos en las que se imparten las asignaturas relacionadas con CaMa, total de horas al semestre, total de créditos otorgados, número de horas teórica, número de horas prácticas, número de horas teórico/prácticas (en caso de no estar desagregada la información) y los objetivos de las unidades académicas relacionadas con el tema de cáncer de mama.

El registro y procesamiento de la información se realizó en Excel®. El análisis de la información se basó en estadística descriptiva, lo que permitió conocer el tipo y frecuencia de temas ofertados relacionados con el estudio del cáncer de mama desde los aspectos puramente biológicos, pasando por los factores clínicos, hasta las acciones en el ámbito poblacional dirigidas particularmente a la promoción de la salud, reconocimiento de signos y síntomas y vigilancia de grupos de riesgo.

Las entrevistas semi-estructuradas (10 en total) a personal académico, se realizaron en cuatro universidades ubicadas en Morelos y en el Distrito Federal. La elección de los informantes fue por muestreo teórico por conveniencia (20). Las entrevistas fueron grabadas previo consentimiento informado, y transcritas literalmente, creándose archivos en Word. Luego se elaboraron matrices con categorías temáticas para el análisis de la información (21).

\section{RESULTADOS}

\section{Entrenamiento de Médicos Generales}

Caracterización de las Facultades/Escuelas de Medicina

Basados en el Catálogo de Carreras deANUIES 2007, la carrera de medicina en México se imparte en 91 Facultades/Escuelas de 68 instituciones de nivel superior; distribuidas en 53 públicas y 38 privadas y se desarrolla 
a través de 60 planes de estudio, los cuales atienden principalmente, las necesidades locales y regionales en la formación de médicos generales.

La organización curricular de los planes de estudio es muy heterogénea. Algunos están desarrollados bajo el sistema de asignaturas, otros por sistemas de módulos y también existen los sistemas mixtos (los que utilizan paralelamente asignaturas y módulos). Así mismo existen diferencias en horas teóricas y prácticas lo que en muchos casos hizo que no fueran comparables. Después de realizar el análisis documental se clasificaron las asignaturas de la siguiente manera:

- De acuerdo al tipo de asignatura. Áreas: biomédica, clínica y sociomédica.

- De acuerdo a la fase de formación. Básica, disciplinar y profesional o terminal (esta última corresponde al internado y al servicio social).

- De acuerdo al carácter de la asignatura. Obligatorias y optativas.

- Se observó que $84,6 \%$ de los programas (77/91) presentan información en sitios Web (47 de instituciones públicas y 30 de privadas). De éstos $42,8 \%$ (33/77) contienen información desagregada de sus planes de estudio por asignaturas/módulos con horas teóricas, prácticas, créditos y 16,8 \% (13/77) con detalles sobre los objetivos, propósitos y características específicas, datos necesarios para responder la pregunta y los objetivos de este estudio.

Formación y entrenamiento de médicos para la atención del cáncer de mama en México. Análisis regional

Las tasas de incidencia y mortalidad por cáncer de mama en el país han tenido un importante incremento en las últimas tres décadas, con una tendencia diferencial por regiones: mayor en el norte y menor en el sur.

Los hallazgos de la comparación de las tasas de mortalidad del cáncer de mama con la oferta educativa para la formación de médicos, es decir, la distribución de Facultades/Escuelas por región geográfica con capacidad para formar este recurso sanitario, se observan en el Tabla 1. Los resultados indican que existe una importante concentración de facultades en el centro del país, sin embargo, en casi todas las entidades federativas se tiene la oferta del programa. 
Tabla 1. Distribución de Facultades/Escuelas de Medicina comparadas con tasas de mortalidad por CaMa por Regiones, México 2007

\begin{tabular}{|c|c|c|c|c|c|}
\hline \multicolumn{2}{|c|}{ Zona "Norte" } & \multicolumn{2}{|c|}{ Zona "Centro" } & \multicolumn{2}{|c|}{ Zona "Sur" } \\
\hline $\begin{array}{c}\text { Tasa mortalidad } \\
\text { CaMa entre } 12,1 \\
\text { y } 18,2 \text { por } 100 \text { mil } \\
\text { Mujeres }\end{array}$ & $\begin{array}{l}\text { No. Instituciones } \\
\text { de Formación en } \\
\text { Medicina }\end{array}$ & $\begin{array}{c}\text { Tasa mortalidad } \\
\text { CaMa entre } 9,6 \\
\text { y } 12,0 \text { por } 100 \\
\text { mil mujeres }\end{array}$ & $\begin{array}{l}\text { No. Instituciones } \\
\text { de Formación en } \\
\text { Medicina }\end{array}$ & $\begin{array}{c}\text { Tasa mortalidad } \\
\text { CaMa entre } 5,4 \\
\text { y } 9,5 \text { por } 100 \text { mil } \\
\text { mujeres }\end{array}$ & $\begin{array}{l}\text { No. Instituciones } \\
\text { de Formación en } \\
\text { Medicina }\end{array}$ \\
\hline Aguascalientes & 1 & Coahuila & 2 & Campeche & 1 \\
\hline Baja California & 5 & D. Federal & 14 & Chiapas & 3 \\
\hline B. California Sur & - & Durango & 3 & Colima & 1 \\
\hline Chihuahua & 2 & Guanajuato & 2 & Guerrero & 1 \\
\hline Jalisco & 7 & Hidalgo & 1 & Michoacán & 1 \\
\hline Nuevo León & 4 & México & 4 & Oaxaca & 2 \\
\hline Sinaloa & 1 & Morelos & 2 & Puebla & 6 \\
\hline Sonora & 1 & Nayarit & 1 & San Luis Potosí & 1 \\
\hline \multirow[t]{4}{*}{ Tamaulipas } & 8 & Querétaro & 2 & Tabasco & 4 \\
\hline & & Zacatecas & 1 & Tlaxcala & 1 \\
\hline & & & & Veracruz & 7 \\
\hline & & & & Yucatán & 2 \\
\hline Total & 29 & Total & 32 & Total & 30 \\
\hline
\end{tabular}

Fuente: Elaboración de los autores con base en el Catálogo de Carreras ANUIES, 2007. Nota: Los estados están ubicados de acuerdo con la distribución de las tasas de mortalidad y por tanto, existen entidades federativas que aparecen en una ubicación diferente a la que le corresponde geográficamente

Tipo de formación que reciben los estudiantes de medicina con relación al cáncer de mama

Todos los planes de estudio de las Facultades/Escuelas de Medicina en México abordan explícitamente temáticas relacionadas con el cáncer desde diversas perspectivas y en sus diferentes ejes curriculares (biomédica, clínica y socio-médica). No obstante, sobre el cáncer de mama son pocas las referencias que se hacen de manera profunda y específica (sólo en 13 planes de estudio).

De manera específica, en los programas analizados se encontraron 49 asignaturas/módulos con objetivos de aprendizaje relacionados con el tema de CaMa en sus diversas etapas y en las diferentes fases de formación y entrenamiento de este personal, las cuales fueron recodificadas con base en los objetivos de aprendizaje. Se concluye que estas unidades didácticas se proponen desarrollar en los estudiantes conocimientos y habilidades que van desde el estudio de las células, pasando por aspectos anatómicos, morfológicos, funcionales, patológicos y clínicos, hasta las acciones comunitarias enfocadas a actividades de identificación de grupos de riesgo, uso de técnicas adecuadas para realizar el examen mamario y la solicitud de 
mastografía y la referencia al especialista, cuando la situación lo requiere (Cuadro 1).

Es importante anotar que una de las limitaciones de este estudio es que no se estimó el tiempo dedicado a la formación y entrenamiento sobre cáncer de mama durante el transcurso de la carrera debido a la forma como se presenta la información de los planes de estudio.

Entrenamiento de Enfermeras licenciadas

El análisis de los programas que se presenta, se centraran sólo en la formación y entrenamiento de las licenciadas en enfermería (formación universitaria) ya que tienen información desagregada por créditos, número de horas por asignatura, objetivos y características de las unidades didácticas.

Caracterización de las Facultades/Escuelas de Enfermería

Basados en el Catálogo de Carreras de ANUIES 2007, se estableció que este programa a nivel de licenciatura se imparte en 108 Escuelas/Institutos pertenecientes a 67 instituciones de nivel superior; distribuidas en 69 públicas y 39 privadas y se desarrolla a través de 43 planes de estudio.

Con relación a los programas analizados, se observa que $88,0 \%$ de ellos $(95 / 108)$ presentan información en sitios Web. De éstos $28,4 \%$ (27/95) contienen información desagregada de sus planes de estudio por asignaturas/ciclos con horas teóricas, prácticas, créditos y 6,3\% (6/95) con detalles sobre los objetivos, propósitos y características específicas; datos necesarios para responder la pregunta y los objetivos de este estudio.

Los programas de enfermería están diseñados en períodos de 8 a 10 semestres, en modalidades escolarizadas y semiescolarizadas y con planes de estudio generalmente agregados en niveles, fases, etapas y ciclos así:

- Etapa básica/ Etapa disciplinaria/Etapa terminal optativa.

- Nivel básico y Nivel formativo o profesionalizante/Optativo.

- Ciclos: Tronco Básico Universitario/Tronco Básico de Área/ Disciplinarias obligatorias/Servicio Social/Optativas libres. 
- Fases: Fase I El hombre como unidad biopsicosocial/Fase II Hombre y comunidades sanos/Fase III Hombres y comunidades enfermos/Fase IV Integrativa.

Cuadro 1. Asignaturas relacionadas con el estudio del cáncer de mama en médicos

\begin{tabular}{|c|c|c|}
\hline $\begin{array}{l}\text { Áreas de } \\
\text { formación }\end{array}$ & $\begin{array}{l}\text { Nombre de las Asigna- } \\
\text { turas (recodificadas) }\end{array}$ & Objetivo relacionado con el cáncer de mama \\
\hline Biomédica & $\begin{array}{l}\text { - Anatomía (general, pa- } \\
\text { tológica, radiológica). } \\
\text { - Biología molecular } \\
\text { - Histología } \\
\text { - Fisiología }\end{array}$ & $\begin{array}{l}\text { Se estudia: } \\
\text { - Forma, volumen, dirección, consistencia, } \\
\text { configuración externa y anatómica de las } \\
\text { mamas. } \\
\text { - Cambios morfo-fisiológicos más frecuentes } \\
\text { que incluyen patologías benignas del seno y el } \\
\text { cáncer de mama. } \\
\text { - Procesos que desencadenan un cuadro } \\
\text { clínico de anormalidad mamaria. }\end{array}$ \\
\hline Clínica & $\begin{array}{l}\text { - Práctica clínica } \\
\text { - Imagenología } \\
\text { - Ginecología y obstetricia } \\
\text { - Medicina interna } \\
\text { - Oncología }\end{array}$ & $\begin{array}{l}\text { - Se enfoca en la práctica del examen clínico } \\
\text { de la mama, } \\
\text { - Se realizan prácticas de interpretación } \\
\text { radiológica: placas de rayos X, ultrasonido, } \\
\text { mamografía y resonancia magnética. } \\
\text { - Se examinan procesos patológicos, tipos } \\
\text { de cánceres más frecuentes y aplican estos } \\
\text { conocimientos en la detección de los más } \\
\text { frecuentes (incluye cáncer de mama). } \\
\text { - Se desarrollan habilidades para establecer un } \\
\text { diagnóstico oportuno y para derivar de manera } \\
\text { correcta al paciente al nivel correspondiente. }\end{array}$ \\
\hline Sociomédica & $\begin{array}{l}\text { - Salud comunitaria } \\
\text { - Salud pública }\end{array}$ & $\begin{array}{l}\text { - Aplicar estrategias de prevención, diagnóstico } \\
\text { y tratamiento del cáncer mamario. } \\
\text { Difundir el programa de detección de cáncer } \\
\text { mamario y capacitar al equipo de salud para } \\
\text { que lo auxilie en estas labores. } \\
\text { - Identificar grupos de riesgo. Realizar técnicas } \\
\text { adecuadas para el examen mamario. Solicitar } \\
\text { cuando se requiera la mastografía. }\end{array}$ \\
\hline
\end{tabular}

Fuente: elaboración de los autores con datos de ANUIES 2007

Los planes de estudio analizados muestran una clara tendencia formativa hacia lo comunitario, es decir, los alumnos poseen las herramientas, conocimientos y habilidades para realizar intervenciones autónomas, interdependientes y dependientes que garantizarán el cuidado a la salud de las personas, la familia y la comunidad.

El número de asignaturas por programa en promedio es entre 15 y 20, las cuales responden a las áreas básicas, disciplinarias y optativas, estas últimas elegidas por los alumnos de acuerdo a sus áreas de interés práctico y en algunos programas llamada área de especialización selectiva que se toma en los últimos dos semestres (VII y VIII). Dentro de ellas están: área de 
cuidados intensivos, administración de servicios de salud, salud comunitaria y línea terminal de obstetricia o de urgencias, entre otros.

Tipo de formación que reciben los estudiantes de enfermería con relación al cáncer de mama

En los programas analizados se encontraron 59 asignaturas/ciclos que tienen relación con el tema de salud de la mama en sus diferentes etapas y en las diferentes fases de la formación de este personal. Dichas unidades fueron asociadas y conjuntadas en 16, utilizando como criterio de agregación los objetivos de aprendizaje. Se concluye que estas unidades didácticas se proponen desarrollar en los estudiantes conocimientos y habilidades muy enfocadas al cuidado individual, familiar y comunitario. Estas se presentan en el Cuadro 2.

\section{DISCUSIÓN}

Aunque los datos disponibles no permitieron al equipo investigador establecer evidencia concluyente, el análisis de los planes de estudio evidencia que en la carrera de medicina se continúa privilegiando contenidos biologistas de carácter curativo/medicalizado (más de tres cuartas partes corresponden a las áreas biomédicas y clínicas), en comparación con las áreas que desarrollan actividades comunitarias (de prevención primaria y secundaria), hallazgos que concuerdan con los resultados de Flores y cols. (22) y Fernández (23). No se encontraron referencias publicadas que nos permitieran comparar los resultados en el tema específico de detección temprana del cáncer de mama en el país.

Sin embargo, los resultados del estudio permiten plantear como hipótesis que este desbalance en la formación del estudiante de medicina (que se convierte posteriormente en el médico general de primer contacto de las mujeres con los servicios de salud, especialmente los públicos) puede contribuir a la escasa detección temprana del cáncer de mama en México, situación que de alguna manera se confirma en el quehacer del médico en los centros de salud de primer nivel: dedican la mayor parte de su tiempo a la consulta médica para resolver problemas particulares de salud y a labores administrativas, entre otras, dejando al personal de enfermería el rol de la promoción de la salud y de los programas preventivos que se deben desarrollar dentro de los programas de la Secretaría de Salud. 
Cuadro 2. Asignaturas relacionadas con el estudio del cáncer de mama en enfermería

\begin{tabular}{|c|c|c|}
\hline $\begin{array}{l}\text { Asignatura } \\
\text { (recodificada) }\end{array}$ & Asignaturas originales & $\begin{array}{l}\text { Objetivo relacionado con el cáncer de } \\
\text { mama }\end{array}$ \\
\hline Anatomía & $\begin{array}{l}\text { - } \quad \text { Anatomía y Salud } \\
\text { - } \text { Anatomía y fisiología } \\
\text { - } \quad \text { Anatomía topográfica } \\
\text { - } \quad \text { Anatomía patológica } \\
\text { Estructura anatómico- } \\
\text { funcional }\end{array}$ & $\begin{array}{l}\text { Se estudia la anatomía y fisiología } \\
\text { del aparato genital femenino. Pelvis } \\
\text { ósea femenina, genitales externos e } \\
\text { internos, mamas. }\end{array}$ \\
\hline Fisiología & $\begin{array}{l}\text { - } \text { Fisiología } \\
\text { - } \text { Fisiología humana } \\
\text { - } \text { Procesos fisiopatológicos } \\
\text { - } \text { Morfofisiología } \\
\text { - Fisiopatología del adulto }\end{array}$ & $\begin{array}{l}\text { Desarrollan los conceptos fisiológicos } \\
\text { de la glándula mamaria, especialmente } \\
\text { sus influencias hormonales ováricas } \\
\text { e hipofisiarias, así como los cambios } \\
\text { que experimenta en determinadas } \\
\text { situaciones como los cambios cíclicos } \\
\text { menstruales durante el embarazo, la } \\
\text { lactancia y la menopausia. }\end{array}$ \\
\hline
\end{tabular}

\begin{tabular}{|c|c|c|}
\hline Propedéutica & $\begin{array}{l}\text { - Propedéutica I } \\
\text { - Propedéutica II } \\
\text { - } \text { Propedéutica de Enfer- } \\
\text { mería } \\
\text { - Enfermería propedéutica } \\
\text { - Práctica de clínicas de } \\
\quad \text { propedéutica }\end{array}$ & $\begin{array}{l}\text { Se capacita para realizar una buena } \\
\text { historia clínica, focalizada en la } \\
\text { patología mamaria con una anamnesis } \\
\text { dirigida hacia los factores de riesgo de } \\
\text { padecer un cáncer de mama. } \\
\text { Desarrolla habilidades para realizar } \\
\text { un examen físico de la glándula } \\
\text { mamaria y de las regiones axilar y } \\
\text { supraclavicular. } \\
\text { Sabe las diferencias entre los } \\
\text { diferentes patrones de la normalidad e } \\
\text { identifica los hallazgos anormales. Así } \\
\text { mismo se capacita para registrar por } \\
\text { escrito dichos hallazgos. }\end{array}$ \\
\hline $\begin{array}{l}\text { Atención } \\
\text { primaria en } \\
\text { salud }\end{array}$ & $\begin{array}{ll}\text { - } & \text { Atención primaria en } \\
\text { salud } \\
\text { - } & \text { Enfermería en atención } \\
\text { primaria } \\
\text { - } \quad \text { Promoción de la salud } \\
\text { individual y familiar } \\
\text { - } \quad \text { Bienestar y promoción } \\
\text { - Salud pública general }\end{array}$ & $\begin{array}{l}\text { Identificar a las mujeres con alto } \\
\text { riesgo de padecer un cáncer de } \\
\text { mama (familiar, genético y personal) } \\
\text { y los procedimientos de su detección } \\
\text { temprana. } \\
\text { Reconocer las principales } \\
\text { enfermedades benignas de la mama, } \\
\text { así como sus diferentes formas de } \\
\text { tratamiento médico y las indicaciones } \\
\text { del tratamiento quirúrgico. } \\
\text { Desarrollar habilidades para la } \\
\text { exploración clínica mamaria. }\end{array}$ \\
\hline $\begin{array}{l}\text { Elaboración } \\
\text { de } \\
\text { Programas de } \\
\text { Salud }\end{array}$ & $\begin{array}{l}\text { Planeación de programas } \\
\text { - } \quad \text { proyectos } \\
\text { Programas de salud }\end{array}$ & $\begin{array}{l}\text { El estudiante deberá aprender a } \\
\text { diseñar y ejecutar programas básicos } \\
\text { de intervención educativa en el primer } \\
\text { nivel de atención, desarrollando } \\
\text { estrategias de promoción y prevención } \\
\text { de la salud integral de la mujer durante } \\
\text { su ciclo vital. }\end{array}$ \\
\hline
\end{tabular}

Fuente: elaboración de los autores con datos de ANUIES 2007 
En la actualidad, basados en las diversas recomendaciones de múltiples organizaciones mundiales sobre la necesidad de transformar los modelos de salud hacia la Atención Primaria en Salud (24, 25), es necesario modificar el modelo educativo predominantemente bio-médico centrado en la enfermedad, la curación y el hospital, reformando los currículos para que se de mayor importancia en los problemas y necesidades de salud de la población. Así mismo, de manera particular, se deberá reforzar la formación de médicos con adecuadas habilidades de comunicación tanto a nivel individual como comunitario, los conocimientos sobre formulación y diseño de planes de educación sanitaria comunitaria y con una formación humanística que le permita desarrollar elementos de responsabilidad social. En este sentido, el entrenamiento que deben recibir los médicos durante su formación profesional para que estén adecuadamente preparados para hacer una detección temprana del cáncer de mama, debe combinar elementos teórico-prácticos, de desempeño y de observancia de las normas éticas y de trato a las personas con dignidad y respeto.

Para las enfermeras, pese a la evidencia encontrada sobre el predominio del estudio de temas relacionados con la comunidad, los planes curriculares parecen tener deficiencias en aspectos puntuales sobre técnicas y procedimientos para realizar una detección temprana del cáncer de mama. En particular, no se encontró explícitamente la enseñanza de la autoexploración mamaria y el examen clínico, lo cual afecta de manera importante el desarrollo de las competencias necesarias para la atención de la población que lo va a requerir. De hecho esta situación en el contexto mexicano, en donde la enfermera generalmente es la responsable de la operación de los programas preventivos (y dentro de ellos los de salud de la mujer) que se desarrollan en los servicios públicos (Secretaría de Salud, Instituto Mexicano del Seguro Social, Instituto de Seguridad y Servicios Sociales de los Trabajadores del Estado), cobra una importancia crítica en términos de formación y entrenamiento.

Finalmente, es importante hacer un llamado a la reflexión a la comunidad académica sobre los contenidos y actividades que se deban estar desarrollando tanto en las escuelas de medicina como de enfermería hoy, ya que diferentes publicaciones establecen que se está sobre-estimulando la utilización de alta tecnología y se está prestando mucha menos atención al desarrollo de las habilidades clínicas de los profesionales, de comunicación 
efectiva y de manejo de las relaciones médico-paciente $(26,27)$. Por otra parte, muchos de los sistemas de salud actualmente se encuentran en fases de revisión, ya que sus modelos de salud con predominancia en lo curativo no han brindado la respuesta integral que requiere la población para sus necesidades de salud. Una mirada al modelo de atención primaria (modelo preventivo) requerirá de profesionales adecuadamente preparados en promoción de la salud y prevención de la enfermedad para afrontar este nuevo reto

Agradecimientos: Las autoras agradecen el apoyo financiero y técnico del Instituto Carlos Slim de la Salud; de la iniciativa Cáncer de Mama Tómatelo a Pecho A.C y de la Fundación Mexicana para la Salud y su Consejo Promotor de Competitividad y Salud. También agradecemos de manera especial a la doctora Felicia Knaul por ser inspiradora del esfuerzo que llevó a la generación de este producto.

\section{REFERENCIAS}

1. Centro para el control y prevención de enfermedades (CDC) [Internet]. Estadísticas sobre el cáncer de mama. Disponible en: http://www.cdc.gov/spanish/cancer/breast/statistics/ Consultado 7 de septiembre de 2010.

2. Garcia M, Jemal A, Ward EM, Center MM, Hao Y, Siegel RL, Thun MJ. Global Cancer Facts \& Figures 2007. Atlanta, GA: American Cancer Society; 2007.

3. Anderson B, Yip Ch, Ramsey S, Bengoa R, Braun S, Fitch M, et al. El Cáncer de Mama en los Países con Recursos Limitados: Sistemas de Atención de Salud y Políticas Públicas. Breast J. 2007; 13(1):S62-S82.

4. Smith RA, Saslow D, Sawyer KA, Burke W, Costanza ME, Evans WP 3rd, et al. American Cancer Society guidelines for breast cancer screening: update 2003. CA Cancer J Clin. 2003; 53:141-169.

5. Kerlikowske K, Grady D, Rubin SM, Sandrock C, Ernster V. Efficacy of screening mammography. A metanalysis. JAMA. 1995; 273(2): 149-154.

6. Kösters JP, Gøtzsche PC. Regular self-examination or clinical examination for early detection of breast cancer. Cochrane Database Syst Rev. 2003; 2:CD003373.

7. Allen T, Van Groningen B, Barksdale D, McCarthy R. The Breast Self-Examination Controversy: What Providers and Patients should Know. J Nurse Pract. 2010;6(6):444-51.

8. Tara S, Agrawal C, Agrawal A. Validating breast self examination as screening modalities for breast cancer in eastern region of Nepal: A population based study Kathmandu Univ Med J. 2008; 6(1): 89-93.

9. Semiglazov VF, Moiseenko VM. Breast self-examination for the early detection of breast cancer: a USSR/WHO controlled trial in Leningrad. Bull World Health Organ. 1987; 65(3): 391-396.

10. Mittra I. Clinical breast examination as a global strategy for screening for breast cancer. Cancer Strategy. 1999;(1):152-156.

11. Mittra I, Baum M, Thornton $\mathrm{H}$, Houghton J. Is clinical breast examination an acceptable alternative to mammographic screening? Br Med J. 2000;321: 1071-1073.

12. The National Archives [Internet]. Delivering primary care. Disponible en: http:// webarchive.nationalarchives.gov.uk/+/www.dh.gov.uk/en/AboutUs/ DeliveringHealthAndSocialCare/TheHealthAndSocialCareSystem/DH_4105335 Consultado 11 enero 2012. 
13. Instituto Nacional de Cancerología. Recomendaciones para la tamización y la detección temprana del cáncer de mama en Colombia. Bogotá: INC; 2006.

14. Knaul F, Nigenda G, Lozano R, Arreola-Ornelas H, Langer A, Frenk J. Breast cancer in Mexico: a pressing priority. Reprod Health Matters. 2008; 16(32):113-123.

15. Secretaría de Salud. Programa de Acción 2007-2012: Cáncer de Mama. México: SSA; 2007.

16. Anderson B, Yip Ch, Ramsey S, Bengoa R, Braun S, Fitch M, et al. El Cáncer de Mama en los Países con Recursos Limitados: Sistemas de Atención de Salud y Políticas Públicas. Breast J. 2007; 13(1):S62-S82.

17. Wall K, Núñez G, Salinas A, Sánchez S. Determinants of the use of breast cancer screening among women workers in urban Mexico. Prev Chronic Dis. 2008;5(2):1-8.

18. Eniu A, Carlson RW, Aziz Z, Bines J, Hortoba'gyi G, Bese N, et al. El Cáncer de Mama en los Países con Recursos Limitados: Tratamiento y Asignación de los Recursos. Breast J. 2007; 13(1):S43-S61.

19. Asociación Nacional de Universidades e Instituciones de Educación Superior. Catálogo de carreras de licenciatura en universidades e institutos tecnológicos. México: ANUIES; 2007.

20. Bertaux, D. Los relatos de la vida en el análisis social. En: Aceves L (Comp). Historia Oral. México: Instituto de Investigaciones José María Mora; 1993.

21. Strauss A, Corbin J. Bases de la investigación cualitativa técnicas y procedimientos para desarrollar la teoría fundamentada. Medellín: Universidad de Antioquia; 2003.

22. Flores $\mathrm{R}$, Sánchez A, Coronado M, Amador JC. La formación médica en México y los procesos en búsqueda de garantizar la calidad de los egresados. Rev Fac Med UNAM. 2001; 44(2):76-80.

23. Fernández JA [Internet]. Las carreras de medicina en México. Perfiles Educativos1996; XVIII. Disponible en: http://redalyc.uaemex.mx/redalyc/src/inicio/ArtPdfRed. jsp?iCve=13207307. Consultado el 11 enero 2012.

24. Organización Panamericana de la Salud. La formación en medicina orientada hacia la Atención Primaria de Salud. Washington, D. C.: OPS; 2008.

25. Organización Panamericana de la Salud/Organización Mundial de la Salud [Internet] $27^{a}$ Conferencia Sanitaria Panamericana. 59a Sesión del Comité Regional. Metas Regionales en materia de Recursos Humanos para la Salud 2007-2015. Washington, D.C., 2007. Disponible en: http://www.paho.org/Spanish/GOV/CSP/ csp27-10-s.pdf Consultado 17 octubre 2010.

26. González J, García J, Mendoza J, Uriega S. La importancia de la investigación en educación médica en México. Rev Med Hosp Gen Mex. 2010; 73 (1): 48-56.

27. Moreno M. Crisis del método clínico. Rev Cubana Med. 1998; 37(2):123-128. 